\title{
Concha bolhosa gigante: uma causa curável de obstrução nasal
}

Ângela Ferreira, ${ }^{1}$ João Carlos Ribeiro, ${ }^{2-3}$ Óscar Barros, ${ }^{4}$ Miguel Silva, ${ }^{2}$ Rui Nogueira, ${ }^{1}$ António Paiva ${ }^{2-3}$

\section{RESUMO}

Introdução: A obstrução nasal é um problema comum na população portuguesa. Influencia negativamente a qualidade de vida e repercute-se na dinâmica familiar. Tem várias causas, entre elas, a concha bolhosa. Os objetivos deste artigo são descrever um caso de concha bolhosa gigante e sintetizar a abordagem ao utente com obstrução nasal nos cuidados de saúde primários.

Descrição do caso: Mulher de 50 anos que recorre ao serviço de urgência por sensação de ouvido tapado à direita. Apresentava também obstrução nasal crónica bilateral, roncopatia e cefaleias. Ao exame objetivo verificou-se otite seromucosa à direita e corneto médio a estender-se para o vestíbulo nasal e também a existência de secreções abundantes e hipertrofia adenoide na nasofibroscopia. Foi medicada com descongestionante nasal e corticóide sistémico e nasal, tendo sido referenciada para consulta interna de otorrinolaringologia. No âmbito desta consulta foi feita biópsia faríngea, que não revelou alterações, e tomografia computorizada dos seios perinasais que evidenciou a existência de uma concha média bolhosa gigante à direita. Realizou tratamento cirúrgico, com resolução da obstrução nasal e das cefaleias e com melhoria da roncopatia.

Comentário: A procura inicial de cuidados de saúde a nível hospitalar ainda existe, como no caso descrito. É importante difundir o papel do médico de família como prestador e gestor de cuidados em diversas áreas.

De modo sistemático, perante queixas de obstrução nasal é fundamental a realização da história clínica e do exame objetivo cuidados. Devem ser pesquisados sinais e sintomas de alarme. Quando, pelo menos, um estiver presente ou perante uma resposta terapêutica insatisfatória deve-se referenciar o utente para consulta hospitalar.

No caso em concreto, a evidência de otite seromucosa unilateral obriga à exclusão de neoplasia da nasofaringe.

As variantes anatómicas constituem causas potencialmente curáveis de obstrução nasal e devem ser consideradas no diagnóstico diferencial da obstrução nasal crónica persistente.

Palavras-chave: Obstrução Nasal; Cornetos.

\section{INTRODUÇÃO}

A obstrução nasal é um problema comum na população portuguesa, com uma prevalência estimada de $34,3 \%$ em indivíduos com idade superior a 14 anos. ${ }^{1}$ Trata-se de um sintoma que influencia negativamente vários aspetos da qualidade de vida, incluindo a qualidade do sono. ${ }^{2}$ Ao traduzir-se numa menor produtividade laboral e escolar e repercutindo-se na dinâmica familiar, ${ }^{1}$ não é raro o médico de família ser confrontado com utentes que apresentem esta queixa, sendo muito importante a sua correta abordagem.

A obstrução nasal tem várias causas (Quadro I). A rinite e a rinossinusite incluem-se nas mais frequentes. ${ }^{3-4}$

1Médico de Medicina Geral e Familiar, USF BRIOSA, ACES Baixo Mondego ${ }^{2}$ Médico, Serviço de Otorrinolaringologia, Centro Hospitalar da Universidade de Coimbra

${ }^{3}$ Médico, Faculdade de Medicina da Universidade de Coimbra

${ }^{4}$ Médico de Medicina Geral e Familiar, CSP Cruz de Celas, ACES Baixo Mondego
Nas causas mais graves, embora raras, encontram-se os tumores e os granulomas. ${ }^{4}$ Entre as variantes anatómicas que cursam com obstrução nasal, destacam-se as conchas bolhosas, que são pneumatizações dos cornetos nasais. ${ }^{5-6}$ Consensualmente, considera-se uma concha bolhosa quando existe pneumatização de, pelo menos, $50 \%$ da altura do corneto médio, avaliada no plano coronal da tomografia computadorizada (TC). ${ }^{7}$ No entanto, alguns autores consideram-na como qualquer grau de pneumatização ${ }^{8}$ e outros apenas quando ambas as porções do corneto médio (lâmina vertical e porção bulbar) estão envolvidas. ${ }^{9}$ Envolve mais frequentemente os cornetos médios, seguidos pelos superiores e, em casos muito raros, pode atingir os inferiores. ${ }^{5-6}$

A concha bolhosa apresenta uma prevalência nos indivíduos com sinusopatia avaliados por TC entre os 24,5 e 44\%.-11 A existência deste intervalo de valores prendese com diferenças nas populações estudadas e nos diferentes critérios utilizados. ${ }^{8-10}$

Em termos clínicos, os indivíduos com esta variante 
QUADRO I. Diagnóstico diferencial da obstrução nasal

\author{
ETIOLOGIA \\ ALTERAÇÕES DA MUCOSA \\ Inflamatórias \\ Rinosinusite (pode ter etiologia infecciosa também) \\ Rinite \\ Pólipo nasal \\ Granulomatose de Wegener, Sarcoidose, Histiocitose \\ Infecciosas \\ HIV, sífilis, tuberculose, vestibulite nasal \\ Medicação \\ Antitiroideus \\ Anti-hipertensores (e.g., $\alpha$ bloqueantes, $\beta$ bloqueantes, \\ inibidores da enzima de conversão da angiotensina, \\ bloqueadores dos canais de cálcio, hidralazina) \\ Alguns antidepressivos \\ Benzodiazepinas \\ Inibidores da 5-fosfodiasterase \\ Estrogénio e progesterona \\ Anti-inflamatórios não esteroides
}

Rinite medicamentosa

\section{ESTRUTURAL}

Anormalidade congénitas

E.g., estenose da abertura piriforme, atrésia coanal, concha bolhosa

Anormalidades adquiridas
Hipertrofia adenóide
Corpos estranhos

Patologia septal (e.g., desvio do septo após trauma, perfuração septal por cocaína intranasal)

Outras (e.g., anormalidade das válvulas nasais, mucocelos)

\section{Tumores}

E.g., carcinoma espinhocelular, papiloma intertido, carcinoma quístico adenoide, neuroblastoma olfativo

\section{CLÍNICA}

Geralmente atingimento bilateral

Esternutos, rinorreia (aquosa ou purulenta), odinofagia, dor facial ou pressão, disosmia, cefaleias.

Esternutos, rinorreia aquosa, prurido, sintomas oculares; os utentes são tipicamente jovens.

Rinorreia, hiposmia/anosmia.

Evolução longa, com sinais sistémicos de doença.

Rinorreia purulenta, cefaleia, por vezes febre.

Atenção aos hábitos medicamentosos; por vezes, dificuldade em estabelecer relação.
Efeito rebound após descongestionantes como oximetazolina ou fenilefrina.

Geralmente atingimento unilateral

Curso crónico.

Geralmente agravamento progressivo e lento.

Rinorreia, roncopatia, respirar com boca aberta.

Geralmente afeta crianças; duração aguda ou subaguda; habitualmente unilateral.

No desvio septal, um lado mais afetado que outro. anatómica geralmente permanecem assintomáticos. ${ }^{11-12}$ No entanto, quando a concha bolhosa é grande e a fossa nasal estreita é causa de obstrução nasal, como no caso descrito de seguida. ${ }^{11}$ Pode também originar rinorreia, roncopatia, cefaleias e febre nas situações em que a mucosa se encontre inflamada. ${ }^{12}$ 
Quanto à terapêutica, nos casos sintomáticos pode-se optar por tratamento médico e/ou cirúrgico. O primeiro, dependendo da etiologia em questão, pode incluir descongestionantes nasais, antibióticos, anti-histamínicos e corticoides nasais. ${ }^{13}$ Porém, só o segundo tipo de tratamento permite uma resolução definitiva da concha bolhosa. A terapêutica cirúrgica está indicada nos casos de obstrução nasal marcada, quando há obstrução do complexo osteomeatal e nas situações em que há doença dos seios perinasais concomitante. ${ }^{13}$ Nos indivíduos assintomáticos não há necessidade de qualquer tratamento. ${ }^{13}$

Os objetivos principais deste artigo são descrever um caso clínico raro de concha bolhosa gigante, alertando para a existência de causas curáveis de obstrução nasal; e sintetizar os principais pontos na abordagem ao utente com obstrução nasal nos cuidados de saúde primários. Com melhores conhecimentos sobre o tema podem ser prestados cuidados de saúde mais ajustados a cada situação, abreviando-se a resolução de sintomas que podem ter impacto negativo importante na qualidade de vida dos utentes.

\section{DESCRIÇÃO DO CASO}

Utente do género feminino, de 50 anos, caucasiana, desempregada, residente em Águeda, inserida numa família unitária. Antecedentes pessoais de tendinite no cotovelo esquerdo, síndroma da coluna vertebral com irradiação e distúrbio ansioso. Sem hábitos medicamentosos, alcoólicos ou tabágicos conhecidos. É de realçar que a utente não apresentava qualquer patologia ou sintoma do foro de otorrinolaringologia (ORL) na lista de problemas dos cuidados de saúde primários. Dos antecedentes familiares destacava-se apenas mãe com história de neoplasia maligna da mama.

A 8 de fevereiro de 2013 recorreu ao serviço de urgência do Centro Hospitalar e Universitário de Coimbra, por sensação de "ouvido tapado" à direita. Em relação ao exame objetivo é de referir: na otoscopia, otite média seromucosa à direita; na rinoscopia anterior, mucosa polipóide e corneto médio a estender-se para o vestíbulo nasal; orofaringe e palpação cervical sem alterações de relevo. Realizou, nessa data, nasofibroscopia, constatando-se secreções mucóides abundantes e hipertrofia adenóide. Foi medicada com corticóide sistémico e nasal e descongestionante nasal, tendo sido referenciada para consulta interna de ORL. No âmbito desta consulta foi realizada biópsia faríngea a 19 de março de 2013, cujo estudo de anatomia patológica foi compatível com hipertrofia adenóide. Ainda no decurso do seguimento apurou-se história de obstrução nasal crónica, bilateral, perene, de agravamento progressivo ao longo dos últimos anos. Referiu também queixas de roncopatia e cefaleias de tensão. Negaram-se outros sintomas, bem como história de traumatismo nasal prévio ou história de atopia. A 15 de abril de 2013 realizou TC dos seios perinasais que evidenciou a existência de uma concha média bolhosa gigante à direita e desvio do septo nasal com convexidade esquerda (Figura 1).

A 12 de dezembro de 2013 foi submetida a cirurgia endoscópica nasossinusal com excisão da lâmina vertical lateral da concha média direita (Figura 2), septoplastia e eletrocauterização dos cornetos inferiores. Um mês depois, a utente encontrava-se sem obstrução nasal, sem cefaleias e com melhoria da roncopatia. Manteve seguimento em consulta externa de ORL até 26 de agosto de 2015, altura em que teve alta, mantendo discretas queixas de roncopatia.

\section{COMENTÁRIOS}

A propósito deste caso abordam-se alguns pontos de reflexão.

Em primeiro lugar, verifica-se com alguma surpresa que a utente não tinha na lista de problemas prévia patologias do foro ORL. Optou por dirigir-se primeiramente aos cuidados de saúde secundários, mais precisamente ao serviço de urgência, a mais de $50 \mathrm{~km}$ da sua residência. As razões para tal não foram apuradas; no entanto, analisando a história apresentada, é natural que para a utente ter optado por uma tão grande deslocação com os respetivos gastos associados, sobretudo em contexto de desemprego, os sintomas terão assumido grande impacto na sua qualidade de vida. É importante continuar a sensibilizar a população para o papel do médico de família como prestador de cuidados de saúde em diversas áreas, estando capacitado para a abordagem inicial das situações mais comuns e para a referenciação aos cuidados de saúde secundários, sempre que se justifique.

Em segundo lugar, e indo de encontro aos objetivos deste trabalho, é importante o médico de família ter presente que, entre as diversas causas de obstrução nasal existem algumas passíveis de cura, como foi evidenciado no caso descrito. De modo sistemático, perante queixas de obstrução nasal, é fundamental a realização da história clínica e do exame objetivo cuidadosos, que só por si fornecem pistas fundamentais para o diagnóstico etiológico. Assim, a obstrução nasal deve ser caracterizada em termos de duração, padrão temporal (nomeadamente se é sazonal, perene ou esporádica), fatores precipitantes e 

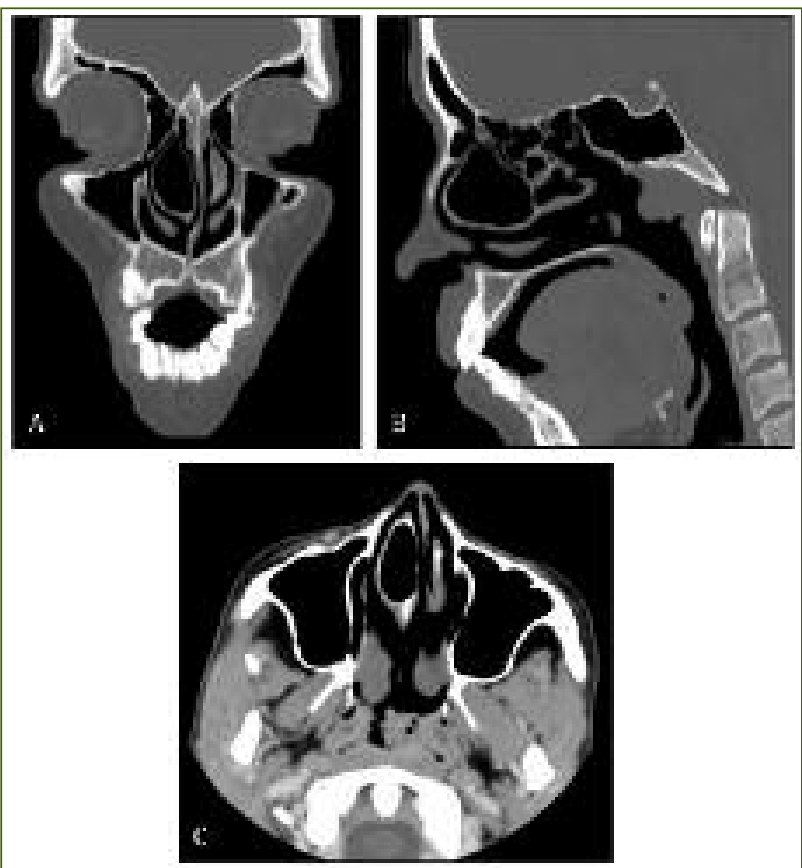

Figura 1. TC seios perinasais - cortes coronal (A), sagital (B) e transversal (C).

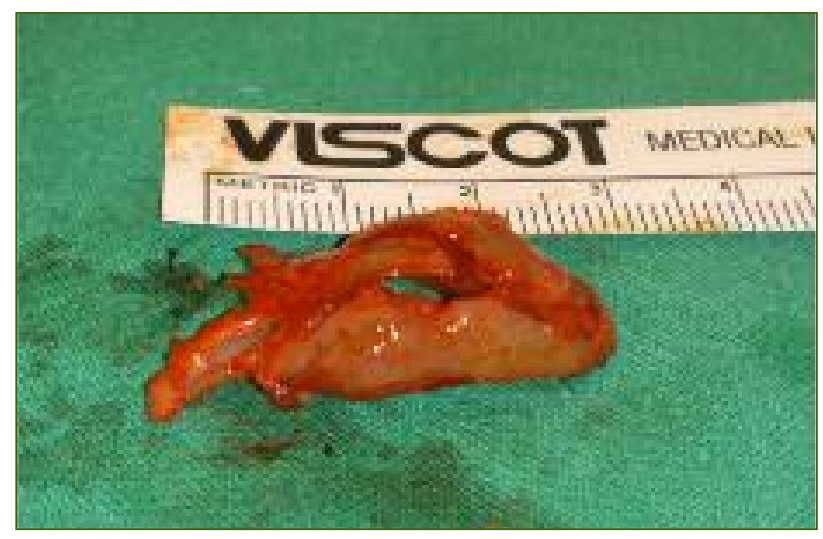

Figura 2. Concha média bolhosa excisada.

se é ou não unilateral. ${ }^{2-4,14}$ Devem ser pesquisados sintomas acompanhantes, entre os quais rinorreia, alterações do olfato, dor facial, cefaleias, prurido nasal, esternutos, sintomas oculares e roncopatia. ${ }^{2-4,14}$ Também tem interesse o conhecimento sobre eventuais traumatismos nasais, tratamentos prévios e respetiva resposta.-4,14 $\mathrm{O}$ exame objetivo deve incluir a avaliação da existência de rinolália fechada e a pesquisa de sinais de atopia (como eczema atópico e dupla prega palpebral). ${ }^{3}$ A pesquisa de sinais e sintomas de alarme é fundamental para exclusão de patologias graves (Quadro II). ${ }^{2,4}$ Quando pelo menos um desses estiver presente deve o utente ser referenciado como prioritário para a consulta de ORL. Também deve ser orientado para consulta hospitalar o utente que não responde de modo satisfatório à terapêutica médica instituída, apesar de adequada compliance. ${ }^{4}$

Em terceiro lugar, referir que no caso concreto, apesar de existir obstrução nasal bilateral, a existência de otite média seromucosa unilateral deve alertar o clínico. Esse diagnóstico torna fundamental a exclusão de neoplasia da nasofaringe. Para tal, é essencial a referenciação precoce para ORL para realização de endoscopia nasal. ${ }^{15}$ Frequentemente é ainda necessário realizar uma biópsia da nasofaringe e uma tomografia computorizada, como foi o caso. ${ }^{15}$

Em suma, as variantes anatómicas, além de frequentes, ${ }^{8}$ constituem causas potencialmente curáveis de obstrução nasal, pelo que devem ser consideradas, sobretudo nos casos de obstrução nasal crónica persistente. Um diagnóstico correto é essencial para uma abordagem terapêutica adequada e eficaz, estando o médico de família numa posição privilegiada para o diagnóstico precoce destas situações.

\section{REFERÊNCIAS BIBLIOGRÁFICAS}

1. Branco-Ferreira M, Morais-Almeida M, Cardoso SM, Barros E, Monteiro L. Congestão nasal em Portugal: epidemiologia e implicações [Nasal congestion in Portugal: epidemiology and implications]. Rev Port Otorrinol Cir Cerv Facial.

\begin{tabular}{l} 
QUADRO II. Sinais e sintomas de alarme num doente \\
com obstrução nasal \\
Unilateralidade da sintomatologia \\
\hline Epistaxes de causa desconhecida \\
\hline Edema palpebral \\
\hline Olho vermelho \\
\hline Proptose ocular \\
\hline Diplopia \\
\hline Oftalmoplegia \\
\hline Redução da acuidade visual \\
\hline Cefaleia frontal uni ou bilateral intensa e única \\
\hline Tumefação frontal \\
\hline Sinais meníngeos ou neurológicos focais \\
\hline Crostas nasais \\
\hline Cacosmia
\end{tabular}


2008;46(3):151-60. Portuguese

2. Van Spronsen E, Ingels KJ, Jansen AH, Graamans K, Fokkens WJ. Evidence-based recommendations regarding the differential diagnosis and assessment of nasal congestion: using the new GRADE system. Allergy. 2008;63(7):820-33.

3. Corey JP, Houser SM, Ng BA. Nasal congestion: a review of its etiology, evaluation, and treatment. Ear Nose Throat J. 2000;79(9):690-8.

4. Krouse J, Lund V, Fokkens W, Meltzer EO. Diagnostic strategies in nasal congestion. Int J Gen Med. 2010;3:59-67.

5. Uzun L, Aslan G, Mahmutyazicioglu K, Yazgan H, Savranlar A. Is pneumatization of middle turbinates compensatory or congenital? Dentomaxillofac Radiol. 2012;41(7):564-70.

6. Souza RP, Brito Júnior JP, Tornin OS, Pais Júnior AJ, Barros CV, Trevisan FA, et al. Complexo nasossinusal: anatomia radiológica [Sinonasal complex: radiological anatomy]. Radiol Bras. 2006;39(5):367-72. Portuguese

7. Stallman JS, Lobo JN, Som PM. The incidence of Concha Bullosa and its relationship to nasal septal deviation and paranasal sinus disease. AJNR Am J Neuroradiol. 2004;25(9):1613-8.

8. Riello AP, Boasquevisque EM. Variações anatômicas do complexo ostiomeatal: achados tomográficos em 200 pacientes [Anatomical variants of the ostiomeatal complex: tomographic findings in 200 patients]. Radiol Bras. 2008;41(3):14954. Portuguese

9. Pérez-Piñas I, Sabaté J, Carmona A, Catalina-Herrera CJ, Jiménez-Castellanos J. Anatomical variations in the human paranasal sinus region studied by $\mathrm{CT}$. J Anat. 2000;197(Pt 2):221-7.

10. Wani AA, Kanotra S, Lateef M,Ahmad R, Qazi SM, Ahmad S. CT scan evaluation of the anatomical variations of the ostiomeatal complex. Indian J Otolaryngol Head Neck Surg. 2009;61(3):163-8.
11. Peri A, Matkovi -Joiin S, Baleti N. Large doubly septated concha bullosa: an unusual anatomic variation. Acta Medica (Hradec Kralove). 2009;52(3):129-31.

12. Yuca K, Kiris M, Kiroglu AF, Bayram I, Cankaya H.A case of concha pyocele (concha bullosa mucocele) mimicking intranasal mass. B-ENT. 2008;4(1):25-7.

13. Cukurova I, Yaz A, Gumussoy M, Yigitbasi OG, Karaman Y. A patient presenting with concha bullosa in another concha bullosa: a case report. J Med Case Reports. 2012;6:87.

14. Bhattacharyya N. Clinical presentation, diagnosis, and treatment of nasal obstruction [internet]. UpToDate [updated 2014 Nov 17; cited 2015 Oct]. Available from: http://www.uptodate.com/contents/clinical-presentation-diagnosisand-treatment-of-nasal-obstruction

15. Alvarenga EH, Maia FN, Souza MA, Cruz OL. Diagnóstico tardio de malformação de tuba auditiva: relato de caso e revisão da literatura [A late diagnose of malformation of ear tube: case report and review of literature]. Rev Bras. Otorrinolaringol. 2003;69(3):421-5. Portuguese

\section{CONFLITO DE INTERESSES}

Os autores declaram não ter conflitos de interesses.

\author{
ENDEREÇO PARA CORRESPONDÊNCIA \\ Ângela Lourenço Ferreira \\ Travessa do Eirô, n. ${ }^{\circ} 7$ \\ Várzea de Calde, 3515-758 Viseu \\ E-mail: angela_ferreira7@hotmail.com
}

Recebido em 07-07-2015

Aceite para publicação em 14-11-2015

\section{ABSTRACT}

\section{GIANT CONCHA BULLOSA: A CURABLE CAUSE OF NASAL OBSTRUCTION}

Introduction: Nasal obstruction is a common problem in Portugal. It can have negative influences on quality of life and repercussions for family dynamics. It has several causes. Among them is concha bullosa. The objectives of this article are to describe a case of giant concha bullosa and to summarize the approach to the patient with nasal obstruction in primary care.

Case description: A 50 year-old woman presented to a hospital emergency department because a blocked right ear. She had experienced chronic bilateral nasal obstruction, snoring and headaches. Right serous otitis media was diagnosed and the middle nasal turbinate was found to extend to the nasal vestibule. There were abundant secretions and adenoid hypertrophy was found on nasal endoscopy. She was treated with corticosteroids and decongestants and referred for otolaryngology consultation. Pharyngeal biopsy was not diagnostic. Computed tomography showed the existence of a right-sided giant concha bullosa. Surgery was performed with resolution of nasal obstruction, relief of headache, and improvement of snoring.

Comment: Some patients may present complaints initially to the hospital emergency department. However it is important to stress the role of the family physician as health care provider and case manager. The initial history and physical examination are important in the investigation of complains of nasal obstruction. 'Red flags' must be investigated. If any of these are present or if there is an unsatisfactory response to medical therapy, the patient must be referred for specialist consultation. In this case, the evidence of unilateral serous otitis media required investigation for the exclusion of nasopharyngeal cancer. Anatomical variants are potentially curable causes of nasal obstruction and should be considered in the differential diagnosis of persistent nasal obstruction.

Keywords: Nasal Obstruction; Turbinates. 